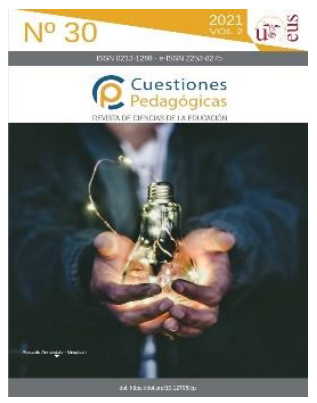

\title{
Educación emprendedora y filosofía de la educación
}

Entrepreneurship education and phylosophy of education

\section{Arantxa Azqueta-Díaz de Alda}

Universidad Internacional de La Rioja

arantxa.azqueta@unir.net

https://orcid.org/0000-0003-2514-5989

\section{Roberto Sanz-Ponce}

Universidad Católica de Valencia

roberto.sanz@ucv.es

https://orcid.org/0000-0003-1147-743X

Resumen: Desde hace unas décadas la educación emprendedora se ha ido incorporado de manera progresiva en el currículo de todas las etapas educativas. Su inclusión es heterogénea y no está exenta de controversias. Uno de los objetivos es asentar y definir el marco teórico para consolidar esta materia en las aulas. El artículo realiza una recopilación de las principales relaciones de la educación emprendedora con las diferentes filosofías de la educación: la educación liberal, la filosofía postestructuralista, el constructivismo y el aprendizaje experiencial. Se emplea un enfoque analítico e interpretativo, fundamentado en la literatura académica. Se concluye que la educación emprendedora no se encuadra con nitidez con una corriente filosófica concreta, porque el interés por esta disciplina nace en el ámbito político y económico y no en el educativo. Además, tanto la filosofía como la tarea emprendedora proceden de ámbitos de conocimiento dispares. Se requiere llevar a cabo una fundamentación antropológica de esta materia que guíe y fundamente los planteamientos pedagógicos del desarrollo humano y no se limite a legitimar
Abstract: For some decades now, entrepreneurship education has been progressively incorporated into the curriculum of all educational stages. Its inclusion is heterogeneous and not without controversy. One of the objectives is to establish and define the theoretical framework to consolidate this subject in the classroom. The article compiles the main relations of entrepreneurial education with the different philosophies of education: liberal education, post-structuralist philosophy, constructivism and experiential learning. An analytical and interpretative approach is used, based on the academic literature. It is concluded that entrepreneurial education does not fit neatly into a specific philosophical current, because the interest in this discipline is born in the political and economic sphere and not in the educational one. Moreover, both philosophy and the entrepreneurial task come from different fields of knowledge. It is necessary to carry out an anthropological foundation of this subject that guides and supports the pedagogical approaches to human development and does not limit itself to legitimize as a subject of educational interest 
como materia de interés educativo lo que goza de racionalidad instrumental o contribuye al progreso de la ciencia y de la tecnología. what enjoys instrumental rationality or contributes

to the progress of science and technology.
Palabras clave: educación emprendedora; filosofía de la educación; aprendizaje experiencial; constructivismo.
Keywords: entrepreneurship education; philosophy of education; discovery learning; constructivism.

\section{Introducción ${ }^{1}$}

El Ya hace algunos años, Hargreaves (2003) meditaba acerca de la dificultad de ser docente en la sociedad del conocimiento. Según el catedrático de la Escuela de Educación y Desarrollo Humano Lynch, del Boston College, el docente se enfrentaba a una profesión "paradójica," porque se le pedía, al mismo tiempo, que preparase para dicha sociedad del conocimiento y para fomentar e incrementar la prosperidad económica -Producto Interior Bruto (PIB)- de los distintos países. Por tanto, debía combatir los excesos de la sociedad del conocimiento: consumismo excesivo, pérdida de sentido de comunidad, ampliación de las diferentes brechas entre ricos y pobres; y a la vez, de alguna manera, también se le pedía que preparase y potenciase estos mismos aspectos: consumismo, globalización, .... Ante esta realidad, proponía formar a los nuevos alumnos en la creatividad y la inventiva, desarrollar en ellos la metacognición, el constructivismo, la comprensión, la neuropedagogía, el aprendizaje cooperativo, las inteligencias múltiples y las tecnologías de la información y de la comunicación. Pretendía crear una nueva identidad cosmopolita y educar la emoción de la empatía. En definitiva, planteaba como meta de la educación superar la sociedad del conocimiento y convertirla en una verdadera sociedad del aprendizaje.

Esta disyuntiva que planteaba Hargreaves (2003) ha sido retomada por otros investigadores de la educación en estos últimos tiempos. Es el caso de autores como Day (2019), Day y Gu (2012), Perrenoud (2006 y 2012), Meirieu (2018), Fullan (2002), Robinson (2015), Hattie (2017), Morín (2016), Pring (2016), Nussbaum (2011), Bauman (2017a y 2017b), entre otros muchos, que se cuestionan acerca del sentido último de la educación. Estos autores denuncian el proceso paulatino de mercantilización de la educación, de instrumentalización de la enseñanza y de su puesta al servicio del mercado. Temen por la actual tendencia social "tecnoeconómica," que "tiende a reducir la educación a la adquisición de competencias socioprofesionales en detrimento de las competencias existenciales"

\footnotetext{
${ }^{1}$ Este artículo está vinculado al proyecto «Formación del Potencial Emprendedor. Generación de un Modelo Educativo de Identidad Emprendedora», con Referencia PID2019-104408GB-I00, dentro del Plan Estatal 2017-2020 de Proyectos I+D+i (Generación de Conocimiento), financiado por: Ministerio de Ciencia e Innovación - Agencia Estatal de Investigación/10.13039/501100011033.
} 
(Morín, 2016, p. 26) y sitúa los conocimientos "calculadores y cuantitativos"2 por encima de los conocimientos "reflexivos y cualitativos." Mantienen que la mentalidad mercantilista ha penetrado y controlado el pensamiento educativo actual, incluso hasta el punto de modificar el vocabulario pedagógico (Pring, 2016). Por ejemplo, en esa misma línea, Perrenoud (2012) encuentra una debilidad en las diferentes reformas educativas. Afirma que estas reformas no se plantean ni cuestionan los contenidos que se deben enseñar en la escuela, que debería ser la primera y más importante de las preguntas ${ }^{3}$, si no, simplemente, se limita a subrayar la importancia de aprender a usarlos y aplicarlos. Esta realidad conduce, también, a los estudiantes a mantener y a adoptar una relación utilitaria con el aprendizaje. Nussbaum (2011) es mucho más tajante en su afirmación. La profesora neoyorquina afirma que la educación se encuentra ante una "crisis silenciosa," ya que se ha rendido ante la lógica y la influencia del mercado. "Producirán generaciones enteras de máquinas utilitarias, en lugar de ciudadanos cabales con la capacidad de pensar por sí mismas, poseer una mirada crítica sobre las tradiciones y comprender la importancia de los logros y los sufrimientos ajenos" (Nussbaum, 2011, p. 20) afirma en uno de sus escritos. Acerca de la "mano invisible del mercado" nos alerta Bauman (2017a, p. 51), que teme que la educación se sitúe solo al servicio del ascenso social, de la garantía del éxito profesional, ..., y olvide los principios y valores esenciales que debe implementar entre el alumnado: el bien común, la democracia, la solidaridad, la profundidad de pensamiento, la creatividad, ... (Bauman, 2017b).

Todo ello, genera un ambiente y un clima donde la educación se mide por el impacto en el Producto Interior Bruto (PIB) y no por el desarrollo personal, moral y social de las personas educadas. Se trata de una mercancía y no de un bien en sí misma. Por ello, todos ellos coinciden en que la educación y la enseñanza deben retomar ese propósito moral, ese propósito que no es otro que el desarrollo personal, moral, social e intelectual de los estudiantes, es decir, prepararlos para la vida en un sentido amplio. Y para conseguir este propósito también se debe replantear las formas de enseñar y de aprender en la escuela. Ante ese caldo de cultivo y en medio de este debate pedagógico, surge la competencia emprendedora con una doble perspectiva o planteamiento filosófico. Por un lado, se encuentra el término enterprise education, que responde a la tradición británica (Erkkilä, 2000), con un significado más amplio y que se refiere al desarrollo de habilidades genéricas, útiles en numerosas situaciones de la vida (Caird, 1992; Hitty, 2008; Pepin, 2012). Por otro lado, se usa el término entrepreneurship education, propio de la tradición americana y que se define como una educación orientada hacia los negocios y la creación de

\footnotetext{
2 El profesor Pring (2016, pp. 15-16) afirma, en ese sentido, que "queda poco espacio para considerar la educación como un factor de transformación de las personas, como el camino que enseña a los jóvenes a ser humanos y se instrumentaliza para servir como medio para conseguir otro fin."

3 “... si hay una "crisis de la enseñanza," no es porque la escuela ha abandonado la transmisión de los saberes... Más bien, hay una crisis del sentido de los saberes, con relación con el saber" (Perrenoud, 2006, p. 94).
} 
empresas (start up, business). Esto genera una gran confusión a la hora de definir y delimitar el marco en el que se encuadra la educación emprendedora en nuestro sistema educativo. También, surge en medio de un debate metodológico y con la intención de plantear una serie de metodologías docentes de carácter activo, experiencial y colaborativo que favorezcan su aprendizaje, y que tiene la intención de impregnar a todo el curriculum académico y a su modo de enseñanza. Por tanto, este artículo se plantea: ¿Cuál de los dos planteamientos filosóficos debe defender, asentar e implementar una educación de carácter general, básica y obligatoria en una sociedad democrática e inclusiva?, ¿qué perspectiva de educación emprendedora debe regir los planes de estudios de la Educación Primaria y de la Educación Secundaria Obligatoria? Y, por último, ¿sobre qué Filosofía de la Educación debe cimentarse una educación emprendedora con un marcado carácter humanista?

\section{Educación emprendedora y filosofía de la educación}

La investigación acerca de la fundamentación teórica de la educación emprendedora es muy escasa. En un estudio de hace unos años que analiza la producción científica de las principales revistas de Filosofía de la Educación de habla inglesa, para el periodo 2000-2010, no se incluye ningún artículo ni se cita a la educación emprendedora explícitamente (Hayden, 2012). Con posterioridad, la investigación científica sobre emprendimiento menciona la escasez de trabajos teóricos que analizan los conceptos educativos del emprendimiento y su relación con los fines de la educación o su relación con las diferentes teorías de la Filosofía de la Educación (Pepin, 2012; Villamor y Prieto, 2014; Prince, Champman y Cassey, 2021). Asimismo, algunos académicos que investigan sobre educación emprendedora han identificado esta necesidad (Bridge, 2017; Gibb, 2011; Hannon, 2005 y 2006; Lackéus, 2016; Taatila, 2010). Bechard y Grégoire (2005) en un estudio centrado en la educación superior señalan que existen pocos académicos que puedan articular este doble conocimiento adecuadamente. Por su parte, para Lackéus (2016) esta ausencia puede estar motivada porque la Filosofía de la Educación y el emprendimiento proceden de ámbitos de conocimiento dispares.

La inclusión de la educación emprendedora como competencia clave en las etapas educativas de Educación Primaria y Educación Secundaria Obligatoria (LOE, 2006; LOMCE, 2013 y LOMLOE 3/2020) imprime a los educadores y a los investigadores educativos la responsabilidad de aportar una fundamentación teórica a la disciplina, de manera que enriquezca el conocimiento y la investigación interdisciplinar sobre el emprendimiento.

En opinión de Bridges (1992), la educación en la iniciativa emprendedora como disciplina específica tiene que vincularse con una filosofía de la educación concreta. Desde el punto de vista del profesorado es también importante fundamentar la tarea docente en una cosmovisión y en una corriente de Filosofía de la Educación. Esto es así por la fuerte repercusión que esta tiene en el estilo de enseñanza e incluso en los propios estudiantes (Ardalan, 2008; Beatty, Leigh y Dean, 2009). 
La educación emprendedora se ha relacionado con las principales escuelas de filosofía de la educación como son la educación liberal, el constructivismo, el aprendizaje experiencial o la filosofía del devenir que se detallan a continuación.

\section{Educación emprendedora y educación liberal}

En primer lugar, algunos autores como Bridges (1992) o Winch y Gingell (2004) buscan relacionar la educación emprendedora con la educación liberal ${ }^{4}$. A través de esta propuesta se busca capacitar a los alumnos para comprender la realidad económica y social y desarrollar sus cualidades personales.

\section{Educación emprendedora y post-estructuralismo}

En segundo lugar, aunque minoritario y en cierta medida novedoso, es la propuesta de Hjorth y Johannisson (2007) que relacionan la educación emprendedora con la filosofía del devenir del filósofo post-estructuralista Gilles Deleuze. Estos autores hacen especial referencia a la naturaleza creativa y relacional de los aprendizajes que proporciona la educación emprendedora.

\section{Educación emprendedora y constructivismo}

En tercer lugar, es más frecuente y resulta mayoritaria en la investigación científica la postura que relaciona la educación emprendedora con la teoría pedagógica del constructivismo (Bruyat, 1993; Bruyat y Julien, 2001; Fletcher, 2007; Gibb, 2011; Kakouris, 2017; Löbler, 2006; Nielsen y Gartner, 2017; Pittaway, 2000; Refai, Klapper y Thompson, 2015; Hägg y Gabrielsson, 2020). Bajo esta perspectiva el proceso de enseñanza y aprendizaje se centra principalmente en el alumno y la motivación tiene un peso importante. Asimismo, el alumno construye su propio proceso de aprendizaje del que es protagonista y la educación da respuesta a necesidades concretas y se adapta al contexto (Löbler, 2006).

\section{Educación emprendedora y aprendizaje experiencial}

Finalmente, otra propuesta mayoritaria es la relación que asocia la educación emprendedora con el aprendizaje experiencial (Caird, 1992; Gibb, 2002; Hitty y O'Gorman, 2004; Johannisson, Landström y Rosenberg, 1998; Pepin, 2012; Pittaway y Cope, 2007; Rae, 2003; Tynjälä, 1999). Este asienta sus presupuestos epistemológicos en la filosofía pragmática que representa John Dewey y que cuenta con un fuerte arraigo en los EEUU, país en el que tiene su origen la formación

\footnotetext{
${ }^{4}$ En el contexto del Reino Unido se entiende por liberal education la tradición educativa con un enfoque humanístico.
} 
empresarial. Para los pragmáticos, la realidad se construye a través de experiencias transaccionales donde los seres humanos interactúan con el medio ambiente, que a su vez cambia constantemente. De este modo, los pragmáticos creen que el conocimiento está sujeto a revisión debido a la naturaleza siempre cambiante del mundo. En ese sentido, los valores son relativos: puesto que la cultura cambia y también lo hacen sus valores (Beatty et al., 2009; Taatila, 2010).

Para Pepin (2012) la filosofía de la experiencia que representa Dewey es de gran valor para la educación empresarial en dos aspectos. El primero, porque establece fuertes vínculos entre las prácticas de enseñanza asociadas con la educación empresarial y la forma en que Dewey (1995) concibe la organización concreta de la educación, a través de lo que denomina "ocupaciones".

Las ocupaciones son actividades sociales básicas que, traídas al aula, proporcionan continuidad entre lo que los estudiantes encuentran dentro y fuera de la escuela. El valor educativo de esas ocupaciones no reside en el carácter formal de las mismas ni en que respondan a los intereses de los estudiantes, sino que su finalidad es educativa y tienen como resultado una actividad de origen social que permite la adquisición de un conocimiento científico. Así, por ejemplo, la jardinería fomentará el interés por la botánica o el papel de la agricultura en la sociedad.

Asimismo, Dewey (1995) resalta la intención transformadora de la realidad que acompaña a toda acción educativa. En esa línea, Altarejos (2006) señala -siguiendo a Polo- la compatibilidad del planteamiento educativo de Dewey con el carácter radical de donación de la persona humana. La educación es un proceso que permite el perfeccionamiento individual y social de las personas y la escuela se entiende como "miniature community, an embryonic society" (Dewey, 1899, p. 15), pero no porque sea una imitación de cualquier tipo de forma política o porque reproduzca la vida en sociedad, sino porque contribuye a la mejora social. Fruto de la naturaleza intrínsecamente social del ser humano es sólo ahí, en la sociedad, donde la persona cumple su misión y su destino. En esta línea introduce un matiz importante: aunque persigue el desarrollo social, este no es solo económico, ni busca la utilidad de este: "el objetivo no es el valor económico de los productos, sino el desarrollo del poder social y su difusión" (Dewey, 1899, p. 16) ${ }^{5}$. El filósofo americano no considera únicamente la mejora del bienestar como tarea imprescindible para la acción educativa, sino que su planteamiento va más allá y considera que se llega a la mejora social a través del perfeccionamiento de las personas, esto es, a través de la adquisición de hábitos y a la formación del carácter, "no podemos pasar por alto los

\footnotetext{
${ }^{5}$ Traducción propia: "The aim is not the economic value of products, but the development of social power and insight" (Dewey, 1899, p. 16).
} 
factores de disciplina y de construcción del carácter involucrados en este tipo de vida" (Dewey, 1899, p. 7) ${ }^{6}$.

En esta línea, considera que la educación en virtudes, especialmente de la sociabilidad y la solidaridad y sus valores afines, contribuyen a formar el compromiso con la transformación social (Dewey, 1899). Bajo el punto de vista de Dewey, el desarrollo de cada persona perfecciona a la sociedad en su conjunto (Guichot, 2003; Naval, 2009; Romo y Nubiola, 2005). Por tanto, la educación es un lugar abierto a la comunidad, que procura dar respuesta a las necesidades sociales y donde no solo se aprenden lecciones. Se destaca la importancia de la labor de adiestramiento que prepara para los deberes prácticos de la vida, porque de esta manera se contribuye a la formación del carácter, en la que el esfuerzo aporta calidad a la educación y a la forja de un espíritu de cooperación social. La noción de progreso deweyana es deudora de la idea de educación que a su vez lleva implícita una antropología y una concepción propia del ser hombre como: "constructor del mundo y donde radica y centra su personalidad" (Guichot, 2003, p. 65). En consecuencia, "las modificaciones que se introducen en los métodos y programas educativos son el producto de los cambios de la situación social y del esfuerzo por satisfacer las necesidades de la nueva sociedad" 7 (Dewey, 1899, p. 4).

También hay que tener en cuenta los trabajos de Kolb $(1984)^{8}$ para quien existe una estrecha relación entre el learning by doing de Dewey (1995) y el ser emprendedor. Kolb entiende el aprendizaje como un proceso de adaptación al mundo, tarea que considera propia de un emprendedor. El aprendizaje experiencial se centra en la importancia de la experiencia en el proceso de aprendizaje, a partir de esta se construye el conocimiento mediante un proceso de reflexión que da sentido a dicha experiencia. Destaca su sentido práctico y su sencillez. Esta metodología invierte la secuencia del aprendizaje tradicional, de la teoría a la práctica, por otra que va de la práctica a la teoría. En ese sentido, esta metodología y forma de entender la educación puede resultar adecuada para introducir el aprender a emprender en la Educación Secundaria Obligatoria y en la Formación Profesional, al involucrar al participante -alumno- en la práctica y hacerle pensar sobre las consecuencias teóricas de las acciones (Lorenzo et al., 2015).

\footnotetext{
${ }^{6}$ Traducción propia: "We cannot overlook the factors of discipline and of character-building involved in this kind of life" (Dewey, 1899, p. 7).

7 Traducción propia: "The modification going in the method and the curriculum is as much a product of the changed social situation, and as much an effort to meet the needs of the new society that is forming" (Dewey, 1899, p. 4).

${ }^{8}$ David Kolb (1984) es un teórico de la educación americano que estudia el aprendizaje experiencial. Desarrolla un Modelo de Aprendizaje Experiencial, Experiential Learning Method (ELM), que se compone de cuatro elementos: experiencia, observación y reflexión de la experiencia, formación de conceptos abstractos basados en la reflexión, experimentación activa de los conceptos nuevos. Estos cuatro elementos forman una espiral de aprendizaje. Realiza un inventario de Estilos de Aprendizaje, Learning Style Inventory (LSI) con cuatro tipos de estudiantes que determina las preferencias de aprendizaje de los individuos.
} 
La idea del "aprender haciendo" la recoge posteriormente la OCDE, que considera como tarea de la escuela no tanto "aprender sobre" sino "aprender a través de", elemento clave en la formación de la tarea emprendedora (OCDE, 2005). Asimismo, coincide con una de las corrientes de educación emprendedora, enterprise education que busca desarrollar en los estudiantes habilidades que pueden ser útiles en numerosas situaciones de la vida (Caird, 1992; Hitty y O'Gorman, 2004). La evolución de la enterprise education ha llevado a diferenciar líneas de trabajo como son la education for enterprise cuyo objetivo es el desarrollo de las habilidades cognitivas que capacitan para pensar, proyectar el futuro, valorar oportunidades, fijar metas y desarrollar la flexibilidad y la education through enterprise que se centra en el desarrollo de las habilidades no cognitivas como son el autocontrol, la perseverancia y la motivación entre otras.

En esa línea, Lackéus (2016 y 2017) es consciente de la necesidad de una filosofía educativa para la educación emprendedora y propone el learning through creating value for others ${ }^{9}$ (p. 80) como el supuesto que relaciona la educación y el emprendimiento y que orienta a los profesores sobre qué hacer, cómo hacerlo y por qué hacerlo. El aprendizaje para la creación de valor para los demás es la pasarela que conecta la educación y la práctica emprendedora. Muchas de las críticas que se oponen o son remisas a la inclusión de la educación emprendedora en el currículo lo hacen porque cuestionan el valor educativo que supone aprender a desarrollar un plan de negocio (Jones y Penaluna, 2013; Neck y Greene, 2011) o porque consideran que promueve la ideología del mercado y del neoliberalismo (Berglund, 2013; Berglund, Hytti y Verduijn, 2020; Bröckling, 2015; Erkkilä, 2000; Fougère, Segercrantz y Seeck, 2017; Gill, 2014; Johannisson, 2010; Komulainen, Naskali, Korhonen y Keskitalo-Foley, 2011; Lackéus, 2017).

\section{Conclusiones}

Se colige en la investigación que la educación emprendedora no se encuadra con nitidez con una corriente filosófica concreta. En primer lugar, el interés por esta disciplina nace, como se ha puesto de manifiesto, en el ámbito político y económico y no en el educativo que lo han convertido en un propósito relevante de instituciones políticas y económicas (Azqueta, 2021). En segundo lugar, su marcado carácter económico dificulta encontrar vínculos con las corrientes de filosofía de la educación puesto que la educación y el emprendimiento proceden de ámbitos de conocimiento dispares.

\footnotetext{
9 Lackéus (2016) emplea el método de investigación abductivo que desarrolla un marco teórico, una investigación empírica y análisis de casos. En el método abductivo la premisa principal resulta evidente pero las premisas secundarias y en consecuencia la conclusión, solo son probables. Aunque se perciba la utilidad y la novedad de una filosofía educativa o pedagógica no implica, sin embargo, que se hayan probado su efectividad en el aprendizaje o que se generalice su práctica entre los profesores.
} 
Es preciso llevar a cabo una fundamentación antropológica que guíe y fundamente los planteamientos pedagógicos del desarrollo humano y no se limite a legitimar como materia de interés educativo lo que goza de racionalidad instrumental o contribuye al progreso de la ciencia y de la tecnología (Musaio, 2013). En ese sentido, una sociedad y una educación excesivamente orientada a la eficiencia y a la utilidad, que limita lo razonable a lo técnico e instrumental, pueden correr el riesgo de deshumanizarse 0 desnaturalizarse. Estas ideas son especialmente consistentes para las etapas de Educación Primaria y Educación Secundaria Obligatoria, en las que se ponen los fundamentos del currículo y que sirven como base de la futura formación profesional. Por ello, de acuerdo con Gimeno Sacristán (2000, p. 33) "la educación práctica o profesional requiere una base cada vez más amplia de formación general que se imparte en la etapa de educación obligatoria." En esa misma línea, Perrenoud (202, p. 14) afirma que el sentido de una educación de carácter integral es permitir a la persona "dar respuesta e intervenir de la manera más apropiada posible con respecto a los problemas y cuestiones que le va a deparar la vida en todos sus ámbitos de actuación." Por tanto, deberíamos ser capaces de implementar una educación técnica, bajo el paraguas de una sólida formación humanista.

Esa es la idea, dar una respuesta los más adecuada posible a todos los desafíos que nos depara la vida, a través del desarrollo de una serie de cualidades y valores que forjan nuestro carácter, que se pretende conseguir con la implementación de la competencia emprendedora, desde una visión humanista y centrada en la persona y su contexto, dentro de los planes de estudio de la educación básica y obligatoria. Se trata como nos dicen Hargreaves y Fullan (2014, p. 216) de decidir entre:

Podemos considerar la enseñanza como una inversión a corto plazo del capital empresarial y financiar el presente hipotecando el futuro de nuestros hijos. $O$ podemos hacer de la enseñanza una inversión sostenible para el capital profesional y dar origen a un mundo de ganancias felices en el futuro.

Entendemos la educación emprendedora como algo más que "hacer negocios" y que supone desarrollar en nuestros alumnos un enfoque más amplio del emprendimiento que supone el cultivo de la identidad emprendedora que promueve la responsabilidad personal y el compromiso activo con la sociedad. Bajo esta perspectiva es deseable no limitar e identificar la educación emprendedora a la educación empresarial. Si la primera se limita a la mejora de las habilidades para la creación de empresas es probable que se convierta en una pedagogía de la empresarialidad (Bernal Guerrero, 2015) y cabría la posibilidad de plantear e impulsar en la escuela una formación especializada en otras opciones profesionales asociadas a otras formas de vida (Barroso, 2015). Nuestra es la decisión y en nuestras manos está el futuro de las siguientes generaciones. 


\section{Referencias}

Altarejos, F. (2006). Estudio introductorio. Leonardo Polo: Pensar la educación. En L. Polo Ayudar a crecer. Cuestiones filosóficas de la educación (pp. 13-39). Ediciones Universidad de Navarra.

Ardalan, K. (2008). The philosophical foundation of the lecture-versus-case controversy: Its implications for course goals, objectives, and contents. International Journal of Social Economics, 35(1/2), 15-34

Azqueta, A. (2021). La educación emprendedora y las instituciones internaciones: respuestas a un desafío global (pp. 35-60). En A. Bernal-Guerrero (ed.). Educación emprendedora. Síntesis.

Barroso, C. (2015). Emprendedor: cuando el término es más que una palabra. En L. Núñez Cuberro (Coord.). Cultura emprendedora y Educación (pp. 133-142). Universidad de Sevilla.

Bauman, Z. (2017a). ¿La riqueza de unos pocos nos beneficia a todos? Paidós.

Bauman, Z. (2017b). Sobre la educación en un mundo líquido. Paidós.

Beatty, J. E., Leigh, J. S. y Dean, K. L. (2009). Philosophy rediscovered exploring the connections between teaching philosophies, educational philosophies, and philosophy. Journal of Management Education, 33(1), 99-114.

Béchard, J. P. y Grégoire, D. (2005). Entrepreneurship Education Research Revisited: The Case of Higher Education. Academy of Management Learning \& Education, 4(1), 22-43.

Berglund, K. (2013). Fighting against all odds: entrepreneurship education as employability training, Ephemera, 13(4), 717-735.

Berglund, K., Hytti U, y Verduijn, K. (2020). Navigating the terrain of entrepreneurship education in neoliberal societies. Entrepreneurship Education and Pedagogy, 3(3), 208-213 https://doi.org/10.1177/2515127420935444

Bernal Guerrero, A. (2015). Sobre la relevancia del factor personal en la investigación en educación emprendedora. En L. Núñez Cuberro (Coord.). Cultura emprendedora y Educación (pp. 127-132). Universidad de Sevilla.

Bridge, S. (2017). Is "entrepreneurship" the problem in entrepreneurship education? Education + Training, 59(7/8), 740-750.

Bridges, D. (1992). Enterprise and liberal education. Journal of Philosophy of Education, 26(1), 91-98.

Bröckling, U. (2015). The entrepreneurial self: Fabricating a new type of subject. Sage.

Bruyat, C. (1993). Création d'enterprise: conntributions épistémologiques et modélisation. Doctoral thesis, Université Pierre Mendès-France-Grenoble II.

Bruyat, C. y Julien, P. A. (2001). Defining the field of research in entrepreneurship. Journal of Business Venturing, 16, 165-180. 
Caird, S. (1992). Problems with the identification of enterprise competencies and the implications for assessment and development. Management and Education Development, 23(1), 6-17.

Day, C. (2019). Educadores comprometidos. Qué son, qué hacen, por qué lo hacen y lo que verdaderamente importa. Narcea.

Day, C. y Gu, Q. (2012). Profesores: vidas nuevas, verdades antiguas. Una influencia decisiva en la vida de los alumnos. Narcea.

Dewey, J. (1899). The School and Social Progress. En The School and Society (pp. 6-30). Chicago University Press.

Dewey, J., (1995). Democracia y educación: una introducción a la filosofía de la educación. Morata.

Erkkilä, K. (2000). Entrepreneurial Education. Mapping the debates in the United States, the United Kingdom and Finland. Garland Publishing.

Fletcher, D. (2007). Social constructionist thinking: some implications for entrepreneurship research education. Handbook of Research in Entrepreneurship Education,1, (pp. 160-172). Edward Elgar Publishing.

Fougère, M., Segercrantz, B. y Seeck, H. (2017). A critical Reading of the European Union's social innovation policy discourse: (Re)legitimizing neoliberalism. Organization, 24(6), 819-843.

Fullan, M. (2002). Las fuerzas del cambio. Explorando las profundidades de la reforma educativa. Akal.

Gibb, A. (2002). In pursuit of a new enterprise and entrepreneurship paradigm for learning: creative destruction, new values, new ways of doing things and new combinations of knowledge. International Journal of Management Reviews, 4(3), 233-269.

Gibb, A. (2011). Concepts into practice: meeting the challenge of development of entrepreneurship educators around an innovative paradigm: The case of the International Entrepreneurship Educators Programme (IEEP). International Journal of Entrepreneurial Behaviour \& Research, 17(2), 146-165.

Gill, R. (2014). If you're struggling to survive day-to-day': class optimism and contradiction in entrepreneurial discourse, Organization, 21(1), 50-67.

Guichot V. (2003). Democracia, ciudadanía y Educación: Una mirada crítica sobre la obra pedagógica de John Dewey. Biblioteca Nueva.

Hägg, G. y Gabrielsson, J. (2020). A systematic literature review of the evolution of pedagogy in entrepreneurial education research. International Journal of Entrepreneurial Behavior \& Research, 26(5), pp. 829-861.

Hannon, P. D. (2005). Philosophies of enterprise and entrepreneurship education and challenges for higher education in the UK. The International Journal of Entrepreneurship and Innovation, 6, pp. 105-114.

Hannon, P. D. (2006). Teaching pigeons to dance: sense and meaning in entrepreneurship education. Education + Training, 48(5), 296-308. 
Hargreaves, A. (2003). Enseñar en la sociedad del conocimiento. Octaedro.

Hattie, J. (2017). "Aprendizaje visible" para profesores. Maximizando el impacto en el aprendizaje. Paraninfo.

Hayden, M. (2012). What Do Philosophers of Education Do? An Empirical Study of Philosophy of Education Journals. Studies in Philosophy and Education, 31, 127.

Hitty, U. (2008). Enterprise education in different cultural settings and at different school levels. En A. Fayolle \& P. Kyrö (Eds) (2008). The dynamics between entrepreneurship, environment and education (pp. 131-148). Edward Elgar Publishing.

Hitty, U. y O'Gorman, C. (2004). What is 'enterprise education'? An analysis of the objectives and methods of enterprise education programmes in four European countries, Education + Training, 46(1), 11-23.

Hjorth, D. y Johannisson, B. (2007). Learning as an entrepreneurial process. En A. Fayolle (Eds.). Handbook of Research in Entrepreneurship Education, (vol. 1), (pp. 46-66). Edward Elgar Publishing.

Johannisson, B. (2010). The agony of the Swedish school when confronted by entrepreneurship (pp 91-106). En K. Kogen y J. Sjovoll (Eds.) Creativity and Innovation. Preconditions for entrepreneurial education. Tapir Academic Press.

Johannisson, B., Landström, H. y Rosenberg, J. (1998): University Training for Entrepreneurship. An Action Frame of Reference. European Journal of Engineering Education, 23(4), 477-496.

Jones, C. y Penaluna, A. (2013). Moving beyond the business plan in enterprise education. Education + Training, 55(8/9), 804-814.

Kakouris, A. (2017). Constructivist Entrepreneurial Teaching: The TeleCC Online Approach in Greece. En P. Jones, G. Maas, L. Pittaway (Eds.) New Perspectives on Entrepreneurship Education. Contemporary Issues in Entrepreneurship Research 7 (235-258). Emerald Publishing Limited.

Kolb. D. (1984). Experiential Learning: Experience as the source of learning and development. Prentice-Hall.

Komulainen, K., Naskali, P., Korhonen, M. y Keskitalo-Foley, S. (2011). Internal entrepreneurship -a Trojan horse of the neoliberal governance of education? Finnish pre-and in-service teachers' implementation of and resistance towards entrepreneurship education, Journal for Critical Education Policy Studies, 9(1), 341-374.

Lackéus, M. (2016). Value Creation as Educational Practice-Towards a new Educational Philosophy grounded in Entrepreneurship? (doctoral dissertation). Goteborg: Chalmers University of Technology.

Lackéus, M. (2017). Does entrepreneurial education trigger more or less neoliberalism in education? Education + Training, 59(6), 635-650.

Ley Orgánica 2/2006, de 3 de mayo, de Educación. BOE, nº 106, 04/05/2005, 1715817207. 
Ley Orgánica 8/2013, de 9 de diciembre, para la mejora de la calidad educativa. $B O E$, $n^{\circ}$ 295, 10/12/2013.

Ley Orgánica 3/2020, de 29 de diciembre, por la que se modifica la Ley Orgánica 2/2006, de 3 de mayo, de Educación. BOE no 340, 30/12/2020.

Löbler, H. (2006). Learning entrepreneurship from a constructivist perspective. Technology Analysis \& Strategic Management, 18, 19-38.

Lorenzo, M., Civila, A., Fernández-Salinero, C. y Naval, C. (2015). Programas de emprendimiento en contextos educativos formales. En L. Núñez Cubero (Coord.) Cultura emprendedora y Educación (pp. 299-325). Ediciones Universidad de Sevilla.

Meirieu, P. (2018). Pedagogía: necesidad de resistir. Editorial Popular.

Morín, E. (2016). Enseñar a vivir. Manifiesto para cambiar la educación. Paidós.

Naval, C. (2009). Educación de la sociabilidad. Ediciones Universidad de Navarra.

Neck, H. M. y Greene, P. G. (2011). Entrepreneurship Education: Known Worlds and New Frontiers. Journal of Small Business Management, 49(1), 55-70.

Nielsen S. L., Gartner W. B. (2017). Am I a student and/or entrepreneur? Multiple identities in student entrepreneurship. Education + Training, 59(2), 135-154.

Nussbaum, M. (2011). Sin fines de lucro. Por qué la democracia necesita de las Humanidades. Katz.

OCDE. (2005). Resumen ejecutivo Proyecto DeSeCo. https://bit.ly/2WctG4e

Pepin, M. (2012). Enterprise education: A Deweyan perspective. Education \& Training, 54(8/9), 801-812.

Perrenoud, P. (2006). El oficio de alumno y el sentido del trabajo escolar. Popular.

Perrenoud, P. (2012). Cuando la escuela pretende preparar para la vida. ¿Desarrollar competencias o enseñar otros saberes? Graó.

Pittaway, L. (2000). The social construction of entrepreneurial behavior (PhD thesis). Newcastle upon Tyne: University of Newcastle upon Tyne.

Pittaway, L. y Cope, J. (2007). Entrepreneurship Education. A systematic review of evidence. Internacional Small Business Journal, 25(5), 479-510.

Prince, S., Champman S. y Cassey, P. (2021). The definition of entrepreneurship: is it less complex than we think? International Journal of Entrepreneurial Behavior \& Research, 27(9), pp. 2647-2673.

Pring, R. (2016). Una filosofía de la educación políticamente incómoda. Narcea.

Rae, D. (2003). Opportunity centred learning: an innovation in enterprise education. Education + Training, 45(8), 542-549.

Refai, D., Klapper, R. G. y Thompson, J. (2015) A holistic social constructionist perspective to enterprise education. International Journal of Entrepreneurial Behavior \& Research, 21(3), 316-337. 
Robinson, K. (2015). Escuelas creativas. La revolución que está transformando la educación. Grijalbo.

Romo, A. P. y Nubiola J. (2005). Virtudes democráticas en la educación de hoy: la propuesta pedagógica de John Dewey. https://bit.ly/3EOluZ9

Taatila, V. (2010). Pragmatism as a philosophy of education for entrepreneurship. En M-L. Neuvonen-Rauhala (Ed.) Innovation and entrepreneurship in universities. The Proceedings of the 3rd International Finnish Network of Entrepreneurship and Innovation for Higher Education (FININ) 2010 Conference, Joensuu, Finland, April 25-27, 2010 (pp. 52-63).

Tynjälä, P. (1999). Towards expert knowledge? A comparison between a constructivist and a traditional learning environment in the university. International Journal of Educational Research, 31(5), 357-442.

Villamor, P. y Prieto, M. (2014). El espíritu emprendedor en educación: un análisis pedagógico. Procedia. Social and Behavioral Sciences, 139, 153-159.

Winch C. y Gingell J. (2004). Philosophy and Educational Policy. A critical introduction. Routledge Palmer. 